\title{
A GENERALIZATION OF CLIFFORD ALGEBRAS
}

\section{by EIFION THOMAS}

(Received 26 December, 1972)

Let $\mathbf{K}$ be a field which contains a primitive $n$th root of unity $\omega$ if $n$ is odd and a primitive $2 n$th root of unity $\zeta$ such that $\zeta^{2}=\omega$ if $n$ is even.

Define $C_{p, q}^{(n)}$ to be the polynomial algebra generated over $\mathbf{K}$ by the set $\left\{e_{1}, \ldots, e_{p}, e_{p+1}, \ldots\right.$, $\left.e_{p+q}\right\}$ subject to the relations

$$
\begin{aligned}
e_{i}^{n} & =+1 \text { for } i=1, \ldots, p ; \\
e_{i}^{n} & =-1 \text { for } i=p+1, \ldots, p+q ; \\
\text { and } e_{i} e_{j} & =\omega e_{j} e_{i} \text { for } 1 \leqq i<j \leqq p+q .
\end{aligned}
$$

$C_{p, q}^{(n)}$ is called a generalized Clifford algebra. Our aim in this paper is to find the structure of $C_{p, q}^{(n)}$ for all values of $p, q$ and $n$. This has already been accomplished for the special cases $p>0, q=0$ and $p=0, q>0$ by A. O. Morris in [1] and [2].

Let $\mathbf{K}(n)$ denote the full matrix algebra of $n \times n$ matrices over $\mathbf{K}$. We first prove

LEMMA 1. There exists an algebra isomorphism

Proof. Define, for $i, j=1, \ldots, n$,

$$
C_{1,1}^{(n)} \cong \mathbf{K}(n)
$$

$$
E_{i j}=\frac{1}{n}\left\{\sum_{p=0}^{n-1} \omega^{(i-1) p} e_{1}^{p}\right\}\left(-e_{2}\right)^{j-i}
$$

As in [1], it can be easily proved that

$$
E_{i j} E_{k l}=\delta_{j k} E_{i l} \text {. }
$$

Let $S=\left\{E_{i j} \mid i, j=1, \ldots, n\right\}$ and put $S_{x}=\left\{E_{i j} \mid j-i \equiv x(\bmod n)\right\}$; then we have $S=\bigcup_{x=0}^{n-1} S_{x}$, $S_{x} \cap S_{y}=\emptyset$ if $x \neq y$.

Since $\omega$ is a primitive $n$th root of unity, we have

$$
\operatorname{det}\left[\omega^{(i-1)(j-1)}\right]=\prod_{0 \leqq i<j \leqq n-1}\left(\omega^{i}-\omega^{j}\right) \neq 0 .
$$

Thus each $S_{x}(x=0, \ldots, n-1)$ is a linearly independent set over $\mathbf{K}$, and therefore so is $S$.

Also $\left(C_{1,1}^{(n)}: \mathbf{K}\right)=n^{2}=(\mathbf{K}(n): \mathbf{K})$ and so the set $S$ is a K-basis for $C_{1,1}^{(n)}$, giving us the required isomorphism.

The next result will enable us to compute inductively the algebras $C_{p, q}^{(n)}$ for any $p, q$ and $n$.

LEMMA 2. There exist algebra isomorphisms

(i) $C_{p, q}^{(n)} \cong C_{1,1}^{(n)} \otimes_{\mathrm{K}} C_{p-1, q-1}^{(n)}$,

(ii) $C_{p, 0}^{(n)} \otimes_{\mathrm{K}} C_{0,2}^{(n)} \cong C_{0, p+2}^{(n)}$,

(iii) $C_{0, q}^{(n)} \otimes_{\mathrm{K}} C_{2,0}^{(n)} \cong C_{q+2,0}^{(n)}$. 
Proof. (ii) and (iii) have been proved in [2, Theorem 4]. For the proof of (i), define

We have

$$
f=e_{1}^{n-1} e_{p+1}^{1-n} \text {. }
$$

If $n=2 d$ is even, then

$$
\begin{aligned}
f^{n} & =\left(e_{1}^{n-1} e_{p+1}^{1-n}\right)^{n} \\
& =\omega^{-\frac{1}{2} n(n-1)^{2}(1-n)} e_{1}^{n(n-1)} e_{p+1}^{n(1-n)} \\
& =\omega^{\frac{1}{2} n(n-1)^{3}} 1 .(-1)^{1-n} .
\end{aligned}
$$

$$
\begin{aligned}
f^{n} & =\omega^{d(n-1)^{3}}(-1) \\
& =-\omega^{d\left(n^{3}-3 n^{2}+3 n-1\right)} \\
& =-\omega^{-d} \\
& =-\omega^{d} .
\end{aligned}
$$

But $\omega^{2 d}=1, \omega^{d} \neq 1$ by the definition of the primitive $n$th root of unity $\omega$, and so

$$
0=\frac{\omega^{2 d}-1}{\omega^{d}-1}=\omega^{d}+1 .
$$

Hence we have $f^{n}=1$ in the case that $n=2 d$ is even.

Similarly, if $n=2 d+1$ is odd

$$
f^{n}=\omega^{n(2 d)^{3} / 2}=\omega^{4 n d^{3}}=1 .
$$

Hence, in either case, we have $f^{n}=1$. Also, for $i=1$ or $p+1$, we have

$$
e_{i} f=\omega f e_{i} \text {. }
$$

Next we define a mapping $\phi$ from $C_{p, q}^{(n)}$ into $C_{1,1}^{(n)} \otimes_{\mathbf{K}} C_{p-1, q-1}^{(n)}$ by

$$
\phi\left(e_{i}\right)= \begin{cases}e_{i} \otimes 1 & \text { if } i=1 \text { or } p+1, \\ f \otimes e_{i} & \text { if } i=2, \ldots, p \text { or } i=p+2, \ldots, p+q .\end{cases}
$$

We have $\phi\left(e_{i}\right)^{n}=1$ for $i=1, \ldots, p$ and $\phi\left(e_{i}\right)^{n}=-1$ for $i=p+1, \ldots, p+q$. Therefore $\phi$ maps identity onto identity. Since $e_{i} f=\omega f e_{i}$ for $i=1$ or $p+1$ and using the defining relations of $C_{p, q}^{(n)}$, we can easily verify that

for $1 \leqq i<j \leqq p+q$.

$$
\phi\left(e_{i}\right) \phi\left(e_{j}\right)=\omega \phi\left(e_{j}\right) \phi\left(e_{i}\right)
$$

Thus, since $\phi$ maps basis elements of $C_{p, q}^{(n)}$ onto basis elements of $C_{1,1}^{(n)} \otimes_{\mathrm{K}} C_{p-1, q-1}^{(n)}$ and

$$
\begin{aligned}
\left(C_{p, q}^{(n)}: \mathbf{K}\right) & =n^{p+q} \\
& =\left(C_{1,1}^{(n)} \otimes_{\mathbf{K}} C_{p-1, q-1}^{(n)}: \mathbf{K}\right),
\end{aligned}
$$

we see that $\phi$ is an isomorphism, as required.

If $A$ is an algebra over $\mathbf{K}$, denote a direct sum of $n$ copies of $A$ by ${ }^{n} A$, i.e.

$$
\left.{ }^{n} A=A \oplus A \oplus \ldots \oplus A \quad \text { ( } n \text { copies }\right) .
$$


The following lemma is [2, Theorem 2].

LEMMA 3. Let $\mathbf{K}$ be a field which contains a primitive nth root of unity $\omega$ if $n$ is odd and a primitive 2 nth root of unity $\zeta$, such that $\zeta^{2}=\omega$, if $n$ is even. Then

(i) $C_{1,0}^{(n)} \cong C_{0,1}^{(n)} \cong{ }^{n} \mathbf{K}$,

(ii) $C_{2,0}^{(n)} \cong C_{0,2}^{(n)} \cong \mathbf{K}(n)$.

Thus we have the following theorem.

THEOREM 4. If $\mathbf{K}$ is a field containing a primitive nth root of unity $\omega$ if $n$ is odd and a primitive 2 th root of unity $\zeta$, such that $\zeta^{2}=\omega$, if $n$ is even, then

(i) $C_{p, q}^{(n)} \cong \mathbf{K}\left(n^{\lambda}\right)$ if $p+q=2 \lambda$ is even and

(ii) $C_{p, q}^{(n)} \cong{ }^{n} \mathrm{~K}\left(n^{\lambda}\right)$ if $p+q=2 \lambda+1$ is odd.

Proof. The proof of both parts of the theorem is carried out by a simple inductive argument using Lemmas 1, 2 and 3.

From now on we shall assume that $K$ does not contain a primitive $2 n$th root of unity $\zeta$ such that $\zeta^{2}=\omega$.

We now define, as in [2], $\mathbf{C}$ to be the quadratic field $\mathbf{K}(\sqrt{ } \omega)$, and $\mathbf{H}$ to be the generalized quaternion algebra regarded as the polynomial algebra over $\mathbf{K}$ generated by $x, y$ subject to the relations

$$
x^{2}=y^{2}=\omega^{-1} .1, \quad x y=-y x .
$$

For completeness, we now state two lemmas which are proved in [2].

Lemma 5. Let $\mathbf{C}$ and $\mathbf{H}$ be defined as above; then there exist isomorphisms

(i) $\mathbf{C} \otimes_{\mathbf{K}} \mathbf{C} \cong \mathbf{C} \oplus \mathbf{C}$,

(ii) $\mathbf{H} \otimes_{\mathbf{K}} \mathbf{C} \cong \mathbf{C}(2)$,

(iii) $\mathbf{H} \otimes_{K} \mathbf{H} \cong \mathbf{K}(4)$.

Proof. This is proved in [2, Lemma 1].

LEMMA 6. Let $\mathbf{K}$ be a field which contains a primitive nth root of unity $\omega$ but not a primitive 2 nth root of unity $\zeta$ such that $\zeta^{2}=\omega$. Then

(i) $C_{1,0}^{(n)} \cong{ }^{n} \mathbf{K}$;

(ii) $C_{0,1}^{(n)} \cong \begin{cases}{ }^{n} \mathrm{~K} & \text { if } n \text { is odd, } \\ { }^{v} \mathrm{C} & \text { if } n=2 v \text { is even; }\end{cases}$

(iii) $C_{2,0}^{(n)} \cong \mathbf{K}(n)$;

(iv) $C_{0,2}^{(n)} \cong\left\{\begin{array}{l}\mathbf{K}(n) \text { if } n \text { is odd or } n=2 v, \text { where } v \text { is even, } \\ \mathbf{H}(v) \text { if } n=2 v, \text { where } v \text { is odd }\end{array}\right.$

(v) $C_{1,1}^{(n)} \cong \mathbf{K}(n)$. 
Proof. (i), (ii), (iii) and (iv) are proved in Theorem 3 of [2].

The proof of ( $v$ ) is exactly the same as in Lemma 1 since the proof did not depend on the existence of a primitive $2 n$th root of unity $\zeta$ such that $\zeta^{2}=\omega$.

We are now in a position to prove

THEOREM 7. If $\mathbf{K}$ is a field which contains a primitive nth root of unity $\omega$ but not a primitive 2 th root of unity $\zeta$ such that $\zeta^{2}=\omega$, then for $n$ odd we have

(i) $C_{p, q}^{(n)} \cong \mathbf{K}\left(n^{\lambda}\right)$ if $p+q=2 \lambda$ is even,

(ii) $C_{p, q}^{(n)} \cong{ }^{n} \mathrm{~K}\left(n^{\lambda}\right)$ if $p+q=2 \lambda+1$ is odd.

Proof. The theorem is proved by a simple inductive argument using Lemmas 1, 2 and 6 . We give the next two results in tabular form.

THEOREM 8. If $\mathbf{K}$ is a field as given in Theorem 7 , then, for $n=2 v$, where $v$ is even, $C_{p, q}^{(n)}$ is given by the table

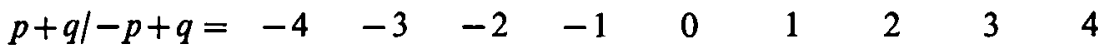

$$
\begin{aligned}
& 0 \\
& 1 \\
& 2
\end{aligned}
$$

Proof. These results follow from Lemmas 2 and 6. For example,

and

$$
\begin{aligned}
& C_{2,1}^{(n)} \cong C_{1,1}^{(n)} \otimes_{\mathbf{K}} C_{1,0}^{(n)}, \quad \text { by Lemma 2(i), } \\
& \cong \mathbf{K}(n) \otimes_{\mathbf{K}}{ }^{n} \mathbf{K}, \quad \text { by Lemma } 6, \\
& \cong{ }^{n} \mathbf{K}(n)
\end{aligned}
$$

$$
\begin{aligned}
C_{3,1}^{(n)} & \cong C_{1,1}^{(n)} \otimes_{\mathbf{K}} C_{2,0}^{(n)}, \quad \text { by Lemma } 2(i) \\
& \cong \mathbf{K}(n) \otimes_{\mathbf{K}} \mathbf{K}(n), \quad \text { by Lemma } 6, \\
& \cong \mathbf{K}\left(n^{2}\right)
\end{aligned}
$$

whereas we have

and

$$
\begin{aligned}
C_{1,2}^{(n)} & \cong C_{1,1}^{(n)} \otimes_{\mathbf{K}} C_{0,1}^{(n)}, \quad \text { by Lemma } 2(\mathrm{i}), \\
& \cong \mathbf{K}(n) \otimes_{\mathbf{K}} \mathbf{C}, \quad \text { by Lemma } 6, \\
& \cong{ }^{v} \mathbf{C}(n)
\end{aligned}
$$

$$
\begin{aligned}
& C_{1,3}^{(n)} \cong C_{1,1}^{(n)} \otimes_{\mathbf{K}} C_{0,2}^{(n)}, \quad \text { by Lemma 2(i), } \\
& \cong \mathbf{K}(n) \otimes_{\mathbf{K}} \mathbf{K}(n) \text {, by Lemma } 6 \text {, } \\
& \cong \mathbf{K}\left(n^{2}\right) \text {. }
\end{aligned}
$$


The remaining entries in the table are obtained in exactly the same way.

THEOREM 9. If $\mathbf{K}$ is a field as given in Theorem 7 and $n=2 v$, where $v$ is odd, then $C_{p, q}^{(n)}$ is given by the table

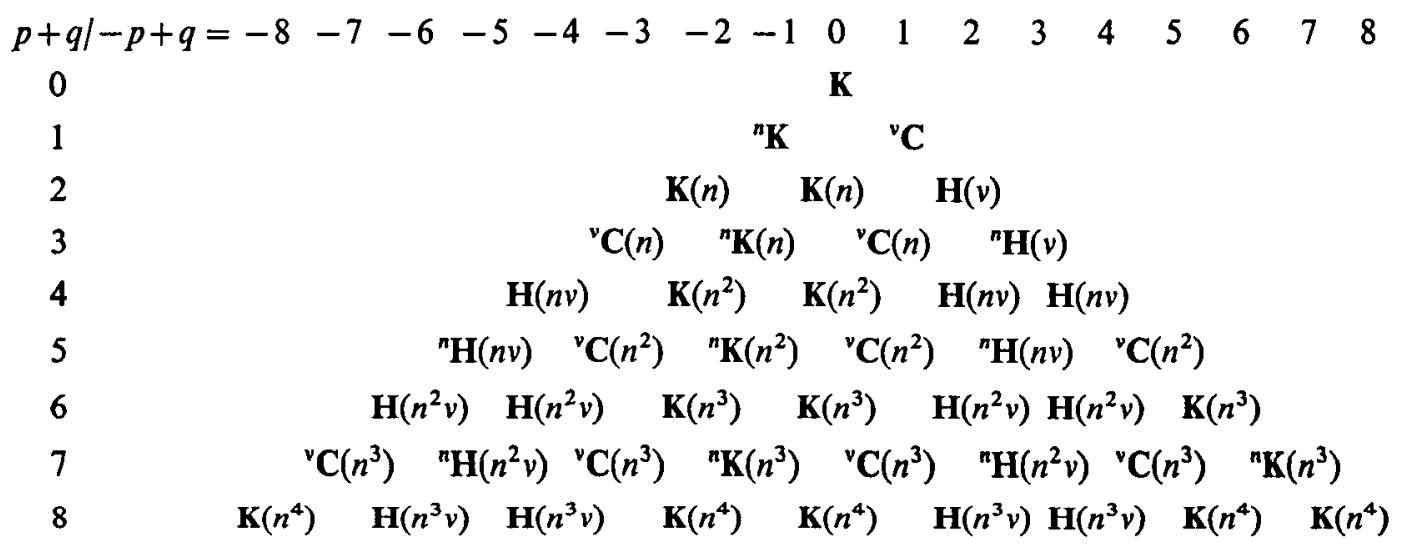

Proof. The theorem follows from Lemmas 2 and 6. We give a couple of examples; the remaining entries in the table are obtained in the same way. For example,

and

$$
\begin{aligned}
C_{3,1}^{(n)} & \cong C_{1,1}^{(n)} \otimes_{\mathbf{K}} C_{2,0}^{(n)}, & & \text { by Lemma } 2(i), \\
& \cong \mathbf{K}(n) \otimes_{\mathbf{K}} \mathbf{K}(n), & & \text { by Lemma } 6,
\end{aligned}
$$

$$
\begin{aligned}
C_{1,3}^{(n)} & \cong C_{1,1}^{(n)} \otimes_{\mathbf{K}} C_{0,2}^{(n)}, \quad \text { by Lemma } 2(i), \\
& \cong \mathbf{K}(n) \otimes_{\mathbf{K}} \mathbf{H}(v), \quad \text { by Lemma } 6, \\
& \cong \mathbf{H}(n v) .
\end{aligned}
$$

We note that the table in Theorem 8 is of periodicity 4 and the table in Theorem 9 is of periodicity 8. These tables have been obtained for the special case $n=2$ in Porteous [3].

\section{REFERENCES}

1. A. O. Morris, On a generalized Clifford algebra, Quart. J. Math. Oxford Ser. (2) 18 (1967), 7-12. 289-299.

2. A. O. Morris, On a generalized Clifford algebra (II), Quart.J. Math. Oxford Ser. (2) 19 (1968),

3. I. R. Porteous, Topological Geometry (New York, 1969).

UNIVERSITY COLLEGE OF WALES

Aberystwyth, CARDiganshiRe 